\title{
Flora de la ciudad universitaria, UNAH: un proyecto de ciencia ciudadana realizado por estudiantes universitarios
}

Lilian Ferrufino, Olvin Oyuela, German Sandoval y Francia Beltrán ${ }^{1}$

\section{RESUMEN}

Las áreas urbanas constituyen sitios fragmentados de los ecosistemas naturales, convirtiéndose en barreras entre las poblaciones de plantas y animales que habitan en ellas. En la ciudad universitaria se han realizado varios inventarios para contribuir a los estudios de impacto ambiental como un requisito para la construcción de edificios en el área, en consecuencia, este estudio tiene como objetivo hacer un inventario florístico del campus.

Para la colecta se estableció la zonificación de la ciudad universitaria: áreas verdes de la parte frontal y central, Palacio de los Deportes, Facultad de Ingeniería y al Observatorio Astronómico. Se colectaron las muestras en flor y fruto durante un año y estas fueron colectadas por los estudiantes de las clases de Taxonomía vegetal I y Taxonomía vegetal II. Se identificaron usando claves dicotómicas, ejemplares depositados en el herbario y con la colaboración de expertos.

Se identificaron cerca 172 especies, pertenecientes a 55 familias, de estas las más comunes son: Fabaceae, Myrtaceae y Asteraceae. Los grupos de plantas que predominan en el área son las angiospermas, con un bajo porcentaje de helechos y gimnospermas. Este estudio servirá de base para proponer un plan de mejoramiento ambiental, ya que la ciudad universitaria constituye uno de los espacios para el conocimiento de la flora urbana de Tegucigalpa. Además, estas acciones contribuirán a impulsar el desarrollo local y la participación de estudiantes universitarios en la investigación científica.

Palabras clave: flora, campus universitario, UNAH, ciencia ciudadana.

\footnotetext{
${ }^{1}$ Profesores universitarios y asistentes técnicos del Herbario Cyril Hardy Nelson Sutherland, Carrera de Biología, Departamento de Biología, Facultad de Ciencias, UNAH: ferrufinolilian@yahoo.com; lilian.ferrufino@unah.edu.hn
} 


\section{ABSTRACT}

Urban areas are fragmented sites of natural ecosystems, with barriers between populations of plants and animals that inhabit them. On campus, there have been several inventories that contribute to environmental impact studies required for the construction of buildings in the area. This study aims to create a floristic inventory of the campus. For the collection, campus zoning was established as follows: green areas of the front and center, Sports Center, Engineering, and the Observatory.

The students of the courses Plant Taxonomy I and Plant Taxonomy II collected the flower and fruit samples during one year. The material was identified using dichotomous keys, specimens in the herbarium and with the collaboration of experts. About 172 species belonging to 55 families were identified.

The most common families are Fabaceae, Myrtaceae and Asteraceae. The plant groups that dominate the area are angiosperms and a low percentage of ferns and gymnosperms. This study will be the basis for proposing a plan for environmental improvement, due to the fact that the campus is one of the spaces for knowledge of urban flora of Tegucigalpa. Furthermore, these actions contribute to the drive of local development and participation of university students in scientific research.

Key words: flora, campus universitario, UNAH, united citizen's science. 


\section{INTRODUCCIÓN}

Los programas de participación ciudadana han jugado un rol muy importante en el estudio de la biología de especies, estudios de ecología, educación ambiental e inventarios. Esta participación se considera un nuevo concepto para personas que participan y contribuyen a realizar estudios científicos durante años.

La escasez de recursos financieros y la acelerada destrucción de los bosques han despertado el interés en estos programas, llevados a cabo por estudiantes universitarios y científicos, voluntarios, de manera que este programa es parte de muchas instituciones educativas, gubernamentales y no gubernamentales (Krasny y Bonney, 2005; Cohn, 2008; Dickinson y otros, 2010; Gardiner y otros, 2012).

Las áreas urbanas constituyen áreas de fragmentación de los ecosistemas naturales, siendo barreras entre las poblaciones de plantas y animales que habitan en ellas (Correa y otros, 2005). En este contexto, en la ciudad universitaria se han realizado varios inventarios para contribuir con los estudios de impacto ambiental como un requisito para la construcción de edificios en el área. House y otros (sin publicar) realizan un inventario arbóreo de tres sitios propuestos para la construcción del futuro edificio de ingeniería dentro de las instalaciones de la UNAH, así como un inventario del complejo polideportivo y del jardín botánico.

El inventario de la flora de la ciudad universitaria tiene como objetivo identificar las especies de plantas vasculares con el propósito de tener un mejor manejo de los recursos. La colección principal consta de una colección de plantas vivas y la segunda consiste en un ejemplar de herbario. Asimismo, en este proyecto se elaboró un catálogo con base en las fotografías de la flora del campus

\section{MATERIALES Y MÉTODO}

\section{Área de estudio}

La ciudad universitaria, UNAH, está ubicada en el bulevar Suyapa, Tegucigalpa, en el departamento de Francisco Morazán, entre las coordenadas de latitud $14^{\circ} 5^{\prime} \mathrm{N}$ y longitud $87^{\circ} 9^{\prime} \mathrm{W}$; a una altura entre $700-900 \mathrm{msnm}$ y con un área de 100 hectáreas aproximadamente. 
Este campus representa un bosque seco subtropical. Se estableció la zonificación del mismo así: áreas verdes de la parte frontal y central, Palacio de los Deportes, Facultad de Ingeniería y Observatorio Astronómico (ver figura 1).

Figura 1. Zonificación de la ciudad universitaria, UNAH

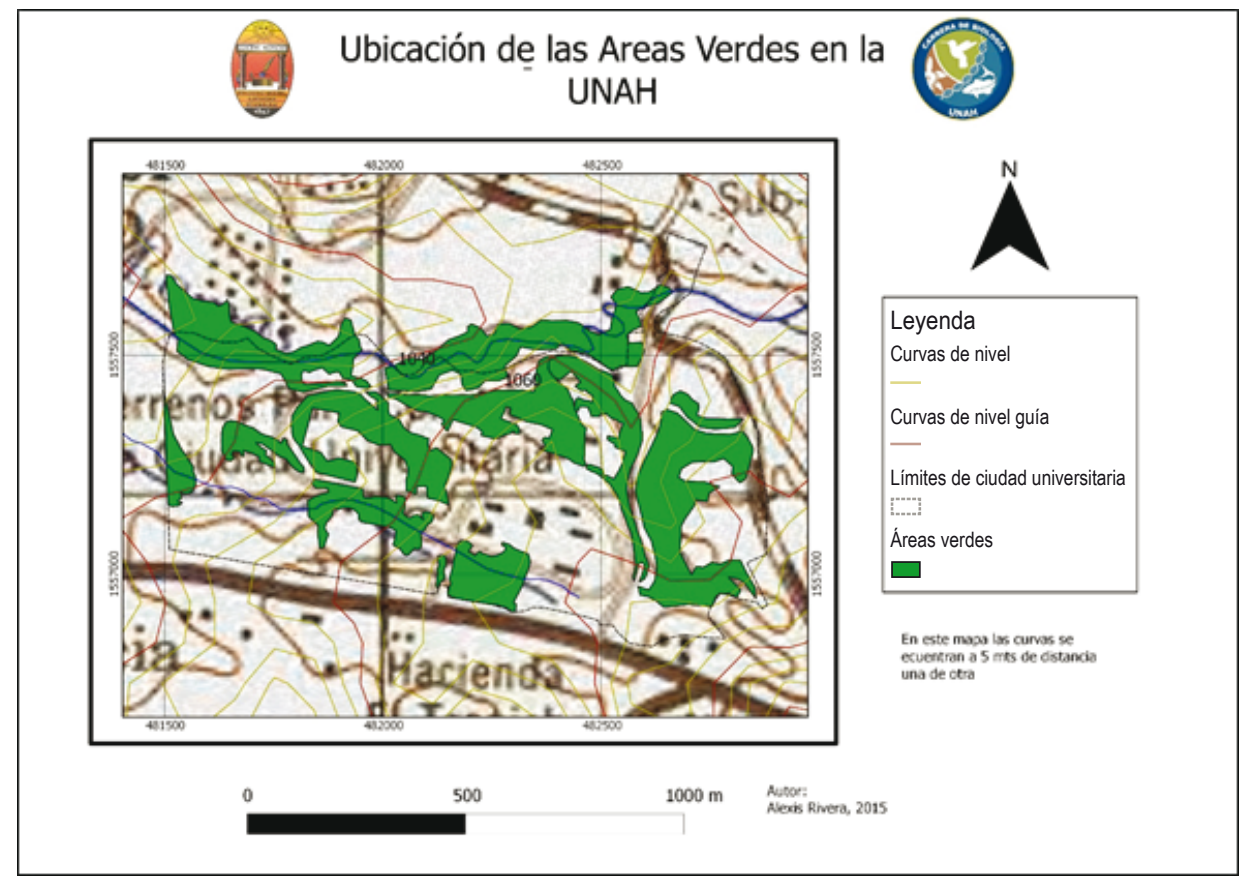

Inventario

Entre 2014-2015 se realizaron colectas de los árboles, arbustos y hierbas que se encuentran en el campus con la colaboración de los alumnos de las asignaturas de Taxonomía vegetal I y Taxonomía vegetal II de la Carrera de Biología. Se tomaron las muestras vegetales con flor y fruto. Estas muestras posteriormente se prensaron (ver figura 2), se les asignó un número de colecta y finalmente se secaron a una temperatura entre los 50 y $70{ }^{\circ} \mathrm{C}$. Las muestras se identificaron por comparación, usando claves dicotómicas y con la ayuda de expertos.

Se elaboraron etiquetas para las muestras botánicas colectadas. Asimismo, se montó este material en pliegos de cartón libre de ácido. Posteriormente, se depositaron en el herbario TEFH y un duplicado fue distribuido en un herbario de la región. Se tomaron fotografías de las plantas con flor y fruto que se encuentran en la colección viva. Los nombres comunes han sido consultados en el catálogo de plantas vasculares de Honduras (Nelson, 2008). 


\section{RESULTADOS Y DISCUSIÓN}

De un total de 172 especies, que representan a 55 familias y 150 géneros (ver cuadro 1 en el anexo), la mayoría de las especies pertenecen al grupo de eudicotiledóneas $(87.7 \%)$, angiospermas basales (2.4\%), monocotiledóneas (7\%), gimnospermas $(1.75 \%)$ y pteridofitas (1.2\%), tal como se muestra en la figura 2 . La mayoría de las especies registradas son arbóreas (46.8 \%), arbustivas (8.6\%), herbáceas $(42.7 \%)$ y lianas (3\%) (ver figura 3). La flora de la ciudad universitaria está constituida por plantas nativas (92 especies) e introducidas (80 especies), como se observa en las figuras $4,5,6,7$ y 8 .

Las diez familias más comunes son: Fabaceae, Asteraceae, Myrtaceae, Solanaceae, Euphorbiaceae, Bignoniaceae, Meliaceae, Malvaceae, Boraginaceae y Arecaceae (ver figura 6). Las especies más comunes pertenecen a la familia Fabaceae, seguidas de Myrtaceae y Asteraceae. La familia Fabaceae representa el grupo de plantas con mayor número de especies en el bosque seco del campus.

Estudios de estructura y composición florística y diversidad en Honduras también han registrado que Fabaceae es la familia más común en los bosques secos, tales como Garcés López (2004) en Masicarán, El Paraíso; Mora y otros (2015) en el valle de Agalta, Olancho; Menéndez Posada y Melara Estrada (2002) en la quebrada Güisisire, Morocelí, El Paraíso.

Figura 2. Número de especies por grupo de plantas

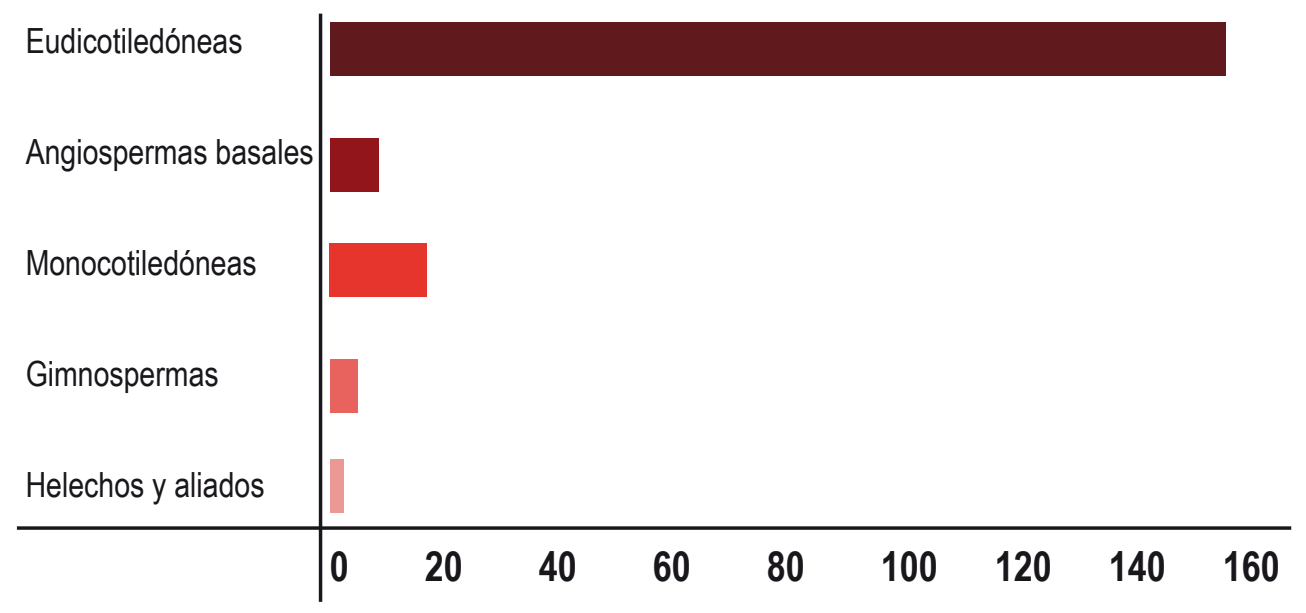


Figura 3. Número de especies según el hábito

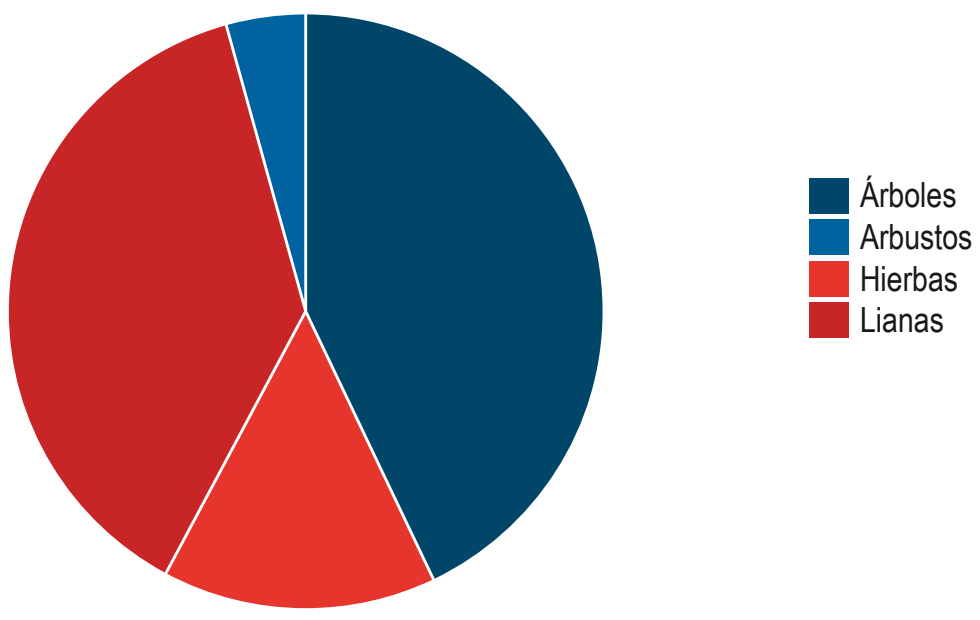

Sin lugar a duda, la ciudad universitaria cuenta con plantas de importancia económica y ecológica, entre las cuales hay plantas comestibles como la anona (Annona muricata), guaba (Inga vera), mamón (Melicoccus bijugatus), limón (Citrus limon), naranja (Citrus $x$ sinensis), granada (Punica granatum), guayaba (Psidum guajava, Psidium friedrichsthalianum), manzana rosa (Syzygium jambos), higo (Ficus pertusa), coyol (Acrocomia aculeata), mango (Mangifera indica), morro (Crescentia alata), níspero (Eriobotrya japonica), jocote (Spondias purpurea) y nance (Byrsonima crassifolia).

Figura 4. Número de especies según origen de las especies

Nativas

Introducidas

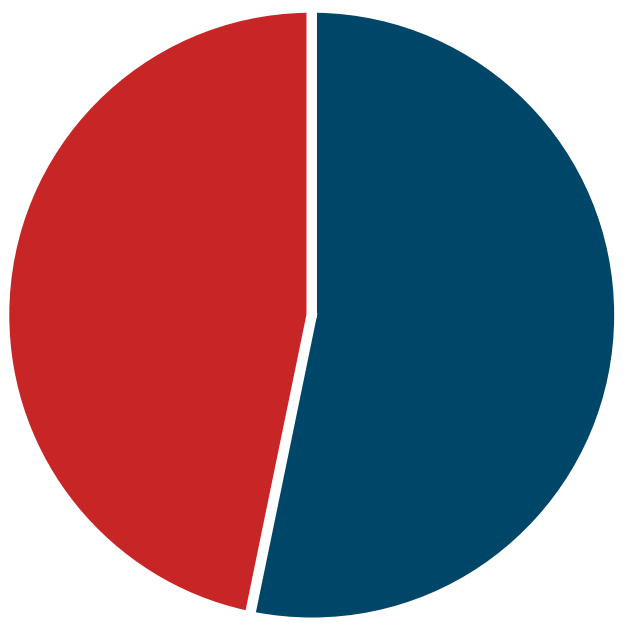


También se registran plantas medicinales, tales como: liquidámbar (Liquidambar styraciflua), espino blanco (Acacia farnesiana), espino negro (Acacia pennatula), eucalipto (Eucalyptus camaldulensis), higuerilla (Ricinus communis), jacaranda (Jacaranda mimosifolia), madriado (Gliricidia sepium), morro (Crescentia alata), cinco negritos (Lantana camara), sábila (Aloe vera). Igualmente se reportan plantas maderables: caoba (Swietenia humillis), cedro (Cedrela odorata), jenízero (Samanea saman), espino blanco (Acacia farnesiana), guanacaste (Enterolobium cyclocarpum), jacaranda (Jacaranda mimosifolia), madriado (Gliricidia sepium), morazán (Delonix regia), pochote (Ceiba aesculifolia), (ver figura 5 y 6 ).

Figura 5. Plantas nativas del campus universitario.
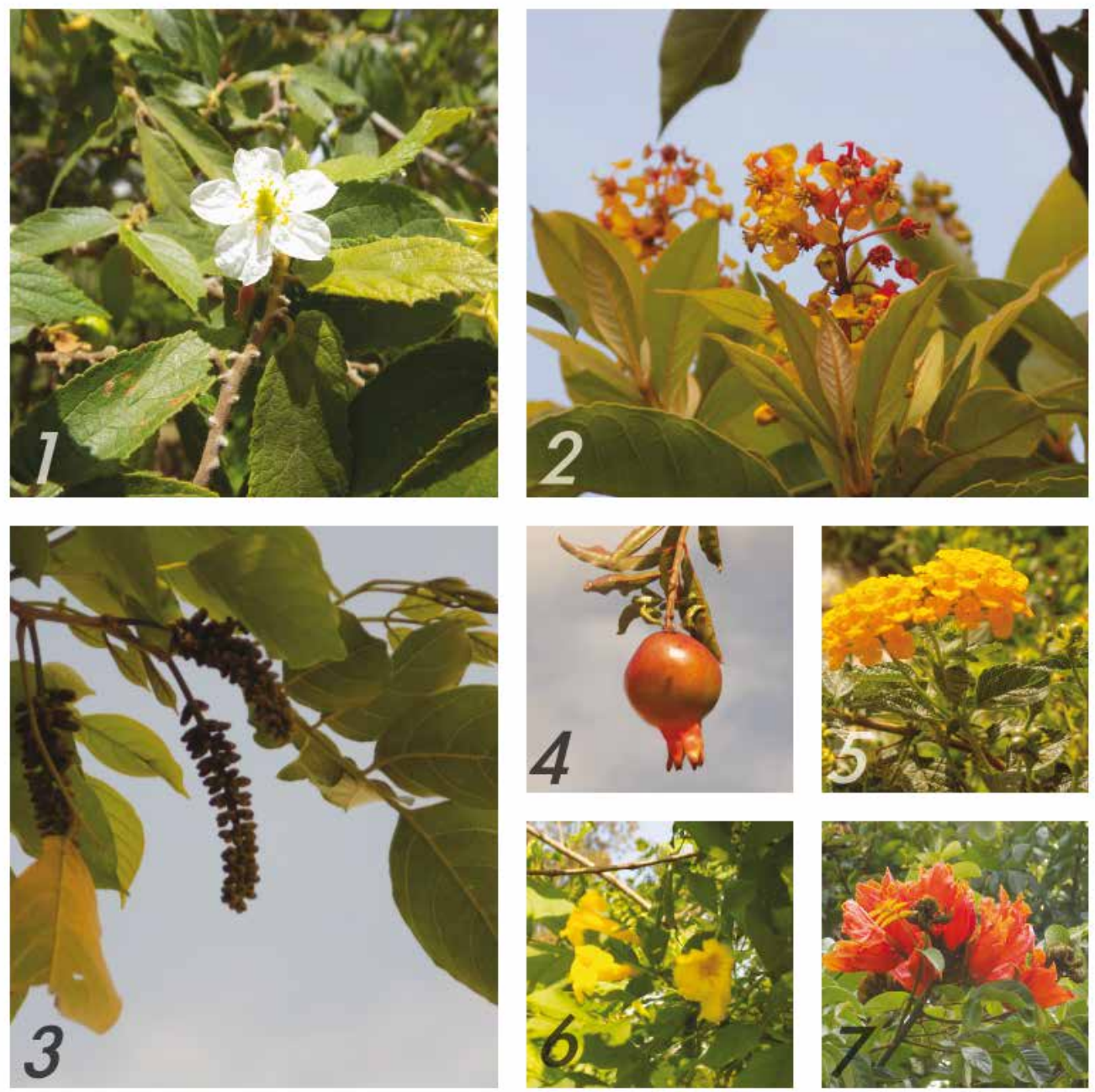

1. Muntigia calabura, 2. Byrsonima crassifolia, 3. Lonchocarpus sanctuarii, 4. Punica granatum, 5. Lantana camara, 6. Tecoma stans, 7. Spathodea campanulata. 
También están presentes plantas ornamentales como: benjamina (Ficus benjamina), casco de burro (Bauhinia divaricata), casuarina (Casuarina equisetifolia), ciprés (Cupressus lusitanica), cinco negritos (Lantana camara), cola de quetzal (Nephrolepis biserrata), corona de Cristo (Euphorbia milii), gravilea (Grevillea robusta), jacaranda (Jacaranda mimosifolia), llama del bosque (Spathodea campanulata), leucena (Leucaena leucocephala), morazán (Delonix regia), napoleón (Boungainvillea spectabillis) y pascua (Euphorbia pulcherrima); las cuales se aprecian en la figura 7.

Figura 6. Árboles del campus universitario.
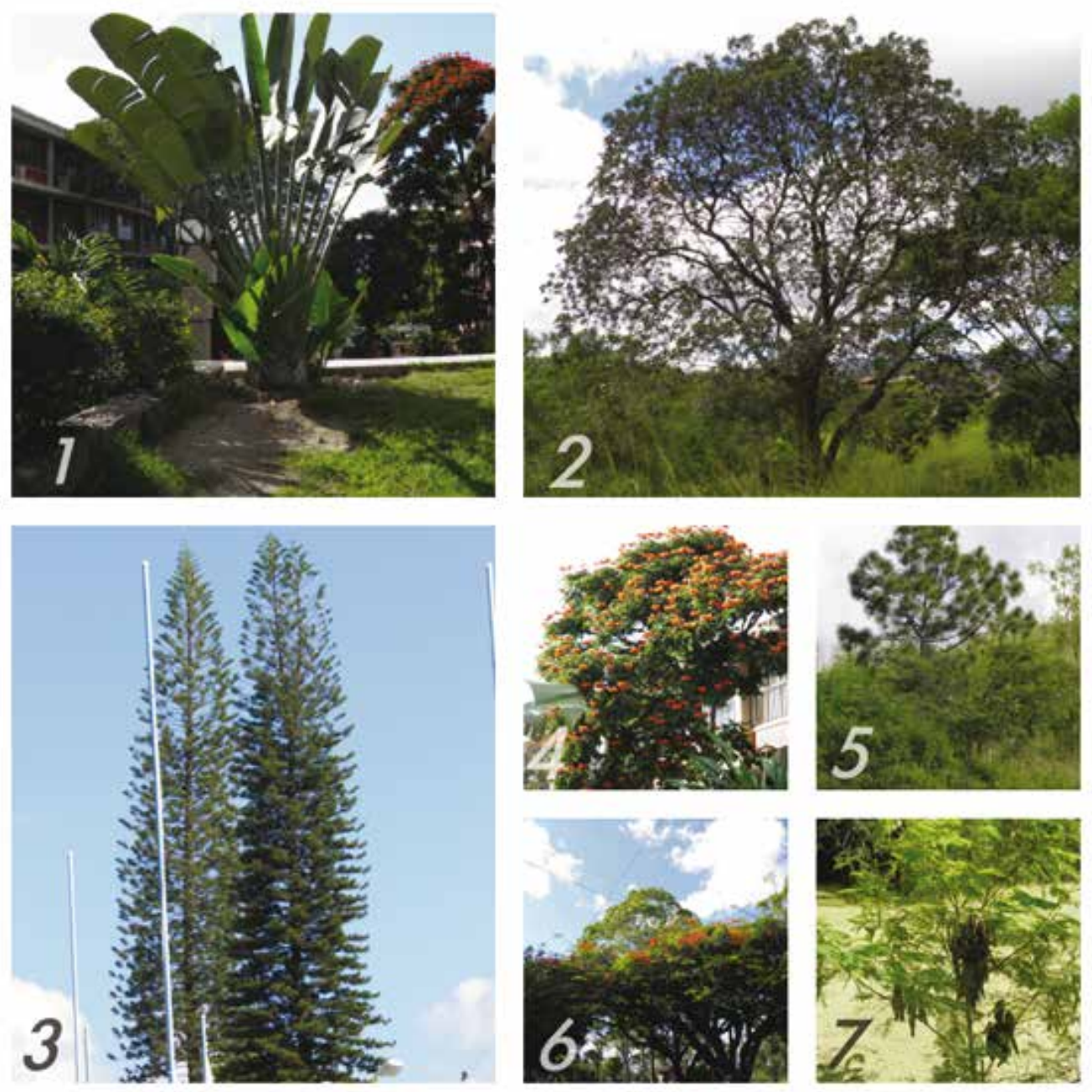

1. Ravenala madagascariensis, 2. Ehretia latifolia, 3. Araucaria heterophyla, 4. Spathodea campanulata, 5. Pinus oocarpa, 6. Delonix regia, 7. Lysiloma auritum 
Figura 7. Plantas cultivadas del campus universitario.
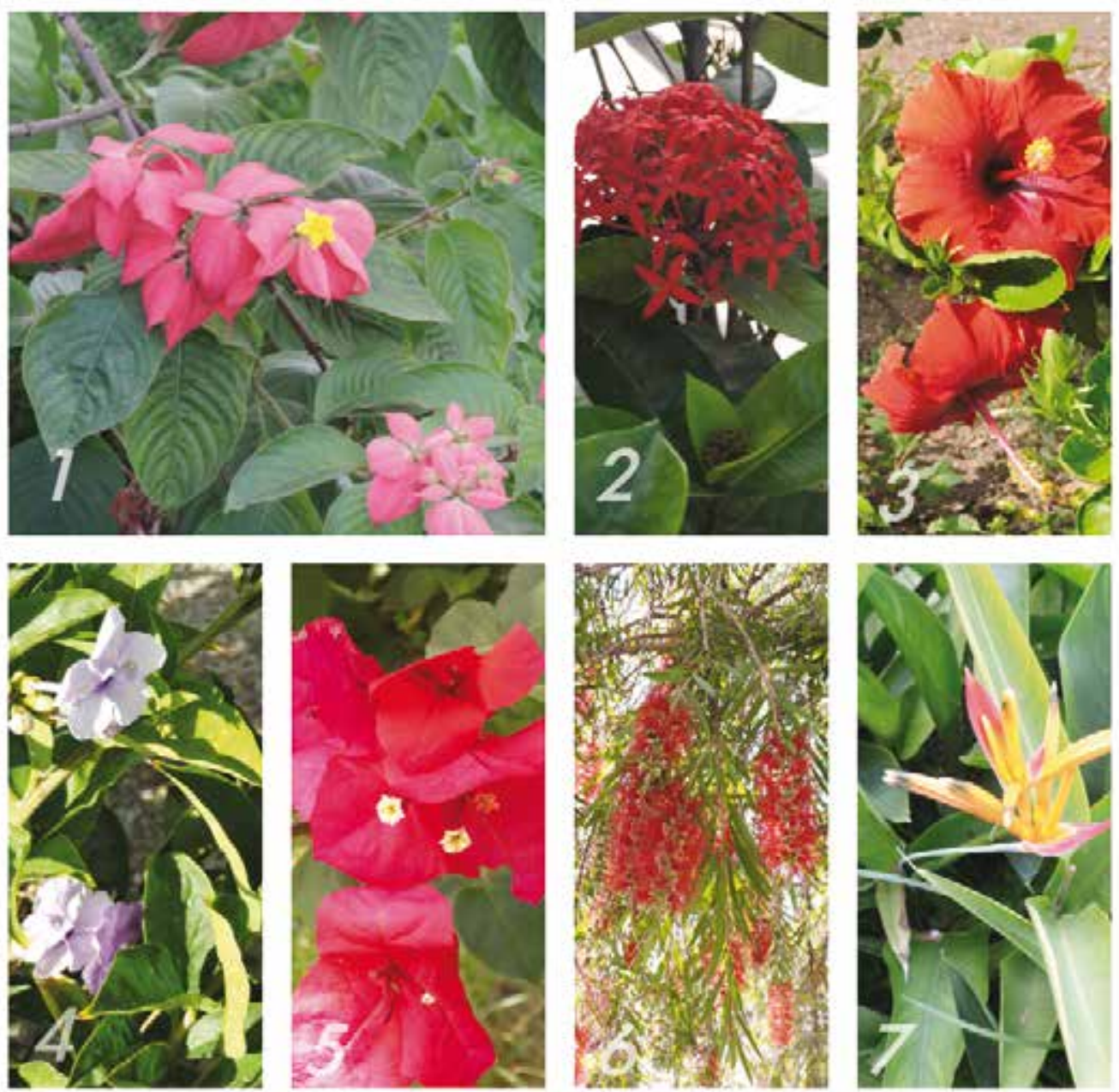

1. Mussaenda erythrophylla, 2. Ixora coccinea, 3. Hibiscus rosa-sinensis 4. Duranta erecta, 5. Bougamvillea spectabilis, 6. Callistemon citrinus, 7. Heliconia psittacorum. 
Figura 8. Plantas nativas del campus universitario.
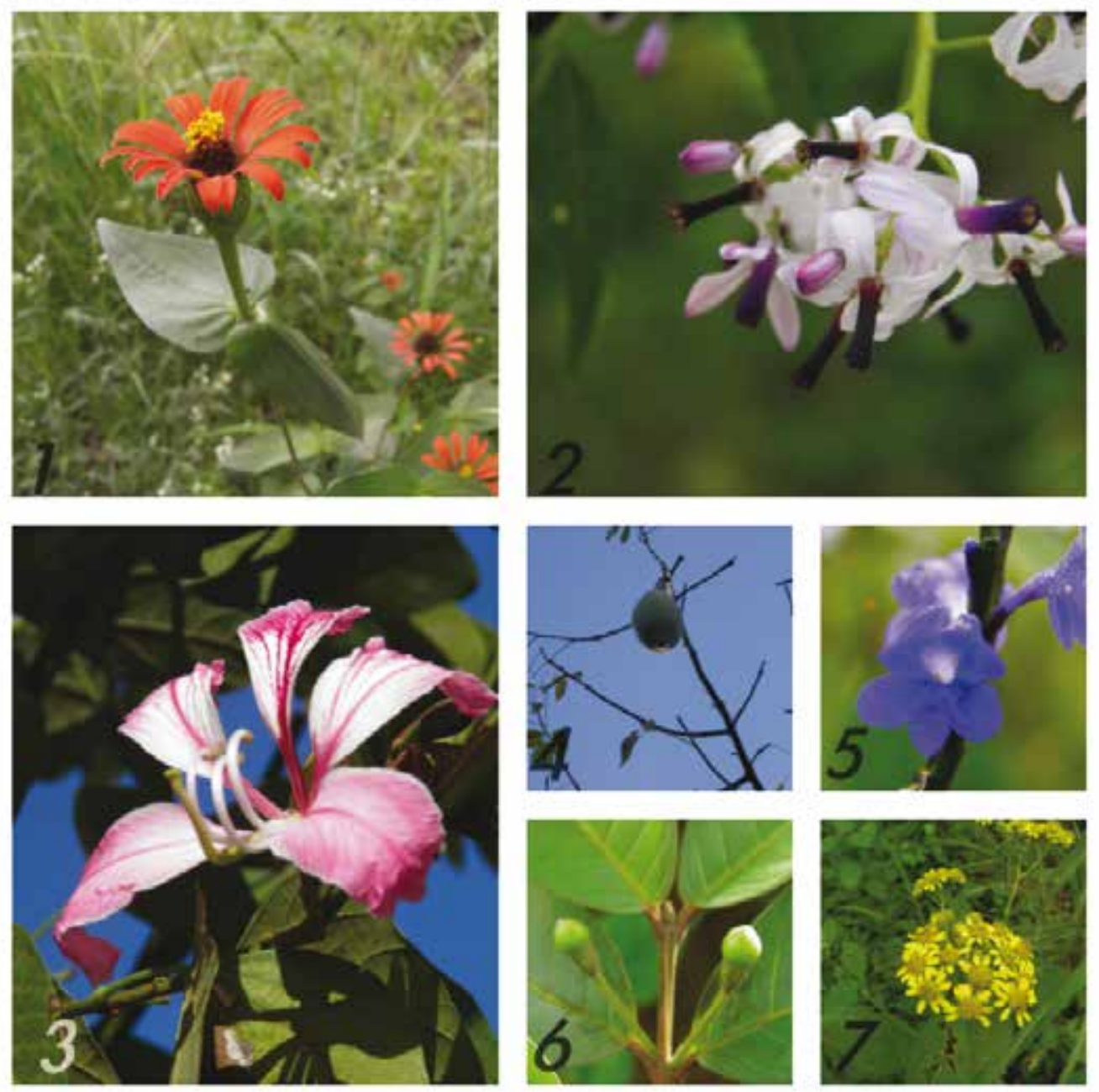

1. Zinnia peruviana, 2. Melia azedarach, 3. Bauhinia divaricata, 4. Ceiba aesculifolia, 5. Stachytharpheta cayennensis, 6. Psidium guianense, 7. Verbesina guatemalensis

Otras plantas son usadas en la elaboración de nidos de pájaro, como la llama del bosque (Spathodea campanulata), sin embargo, en el campus se registra la presencia de la planta invasora Eichhornia crassipes, llamada comúnmente lirio de agua, que ha sido introducido en las represas en Honduras para fitorremedación; así como los zacates Melinis repens, Hyparrhenia rufa y Urochloa máxima. 
Figura 9. Plantas cultivadas del campus universitario.
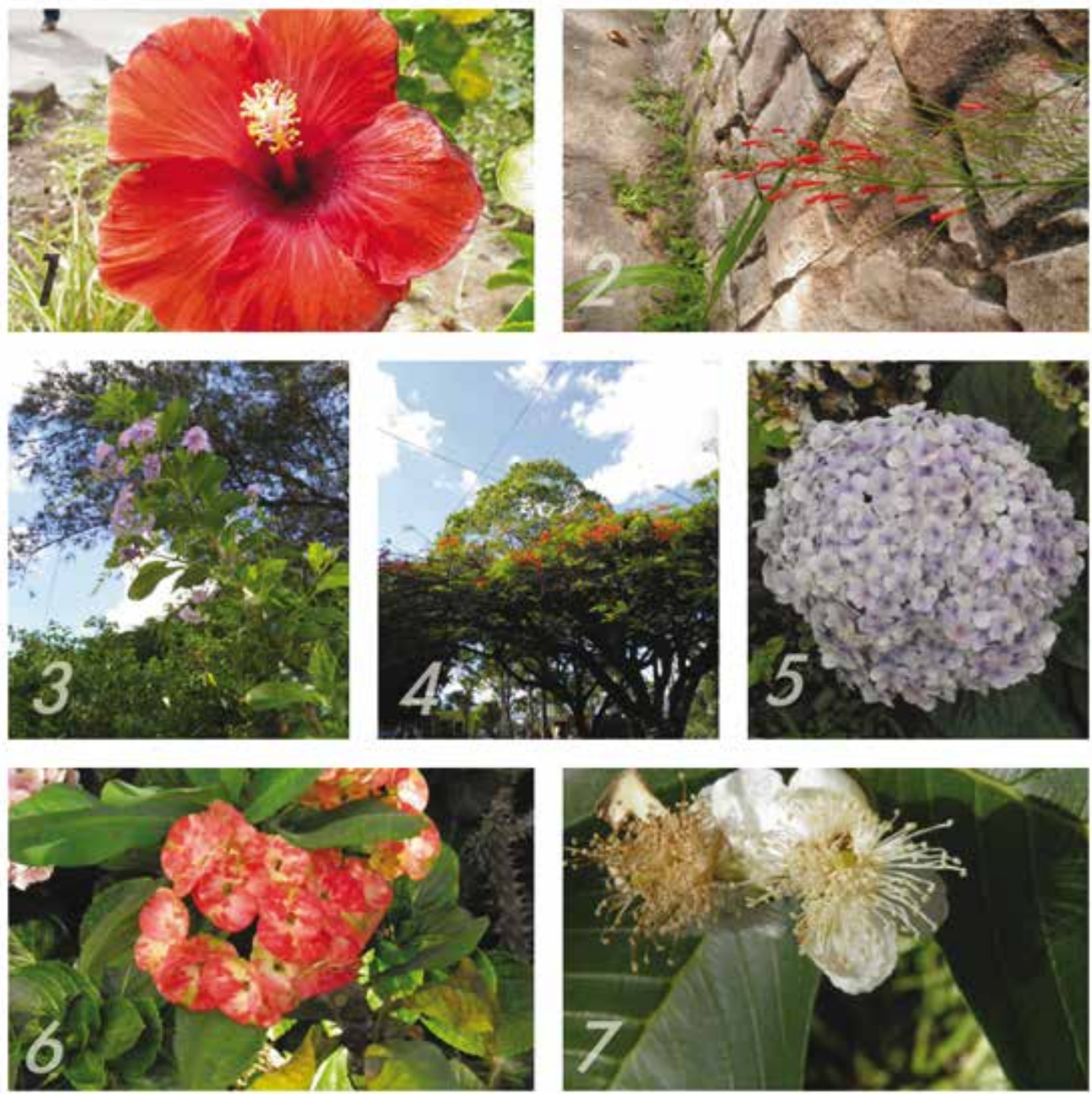

1. Hibiscus rosa-sinensis, 2. Russelia equisetiformis, 3. Duranta erecta, 4. Delonix regia, 5. Hydrangea spp., 6. Euphorbia milii, 7. Psidum guajava.

En la lista roja de especies UICN se registran como especies vulnerables el cedro (Cedrela odorata) y la caoba (Swietenia humilis). Según CITES, ubica estas dos últimas especies en el apéndice III, que incluye a las especies que están protegidas en más de un país y en donde se solicita colaboración de otros países para controlar el comercio.

Muchos inventarios de plantas se han realizado en campus de universidades y se han elaborado listados de plantas georreferenciadas, catálogos y bases de datos, como 
el caso de la Universidad de San Diego, Florida; en Gainesville, Ohio y Luisiana en Lafayette, entre otros; no obstante, en las áreas verdes de las ciudades se registran especies introducidas (Correa y otros, 2005). En síntesis, estos estudios han contribuido a la conservación y manejo de la flora urbana a través de la investigación, educación y participación de la comunidad.

\section{CONCLUSIONES}

Las especies más comunes pertenecen a la familia Fabaceae en este ecosistema. En la ciudad universitaria se registran especies medicinales, ornamentales, maderables y comestibles; entre estas especies se reportan dos especies maderables vulnerables: Cedrela odorata y Swietenia humilis; así como una especie en peligro crítico: Lonchocarpus sanctuarii. La mayoría de las especies son angiospermas con un hábito arbóreo o herbáceo. Estas especies son nativas o introducidas.

No cabe duda que la participación de los catedráticos, asistentes técnicos y estudiantes de la Carrera de Biología en el estudio de la flora de la ciudad universitaria será la base para proponer un plan de mejoramiento ambiental, ya que este campus constituye uno de los espacios para el conocimiento de la flora urbana de Tegucigalpa. Además, estas acciones contribuirán a impulsar el desarrollo local y la participación de estudiantes universitarios en la investigación científica.

\section{AGRADECIMIENTOS}

A los estudiantes de la asignatura de Taxonomía vegetal I y Taxonomía vegetal II, del I periodo de 2014 y 2015. También se agradece a Alexis Rivera por la elaboración de mapas y a los revisores anónimos por su colaboración. 


\section{BIBLIOGRAFÍA}

Convention on International Trade in Endangered Species of Wild Fauna and Flora (CITES). Apéndices I, II \& III (s.f.). Recuperado: https://www.cites.org Cooper, C. B.; Dickinson, J.; Phillips, T. \& Bonney, R. (2007). Citizen science as a tool for conservation in residential ecosystems. Ecology and Society, 12(2), 11. Recuperado de: http://www.ecologyandsociety.org.

Correa M., M.A.; Trujillo, T.E. \& Frausin, B.G. (2005). Inventario de la flora del campus de la Universidad de la Amazonía, municipio de Florencia (Caquetá - Colombia). Momentos de Ciencia, 2(2), 107-115.

Dickinson, J. L.; Zuckerberg B. \& Bonter, D. N. (2010). Citizen Science as an Ecological Research Tool: Challenges and Benefits. Annual Review of Ecology, Evolution and Systematics, 41, 149-172.

Garcés López, P. Á. (2004). Composición florística del bosque seco Masicarán, valle de Yeguare, Honduras, C.A. Tesis. UNAH. Tegucigalpa.

Gardiner, M. M.; Allee, L.L.; Brown, M.J.; Losey, J. E.; Roy, H. E. \& Smyth, R. R. (2012). Lessons from lady beetles: accuracy of monitoring data from US and UK citizen-science programs. Frontiers in Ecology and the Environment, 10, 471-476. Recuperado de: http://dx.doi.org/10.1890/110185

Menéndez Posada, R. N. y Melara Estrada, N. W. (2002). Composición florística del bosque de Galería de la Quebrada, Güisisire, Morocelí, El Paraíso, Honduras, C.A. Tesis. UNAH. Tegucigalpa.

Mora, J. M.; Espinal, M. R.; López, L. I. y Quezada, B. O. (AÑO). Caracterización del bosque seco tropical remanente en el valle de Agalta, Honduras. La Ceiba, 53(1), 38-56.

Nelson, S. (2008). Catálogo de las plantas vasculares de Honduras: espermatofitas. Tegucigalpa: Guaymuras y Secretaría de Recursos Naturales y Ambiente.

UICN. The IUCN Red List of Threatened Species. Recuperado: http://www.iucnredlist.org 


\section{Anexo}

Lista de especies registradas en ciudad universitaria

\begin{tabular}{|c|c|c|c|c|c|}
\hline No. & Familia & Especie & Origen & Nombre común & Hábito \\
\hline 1 & Altingiaceae & Liquidambar styraciflua L. & $\mathrm{N}$ & Liquidámbar & A \\
\hline 2 & Amaranthaceae & Amaranthus hybridus L. & $\mathrm{N}$ & $\begin{array}{l}\text { Bledo manso, moco de } \\
\text { pavo }\end{array}$ & $\mathrm{H}$ \\
\hline 3 & Anacardiaceae & Anacardium occidentale L. & I & Marañón & A \\
\hline 4 & Anacardiaceae & Spondias purpurea L. & $\mathrm{N}$ & Jocote, ciruela & A \\
\hline 5 & Anacardiaceae & Mangifera indica L. & I & Mango & $A$ \\
\hline 6 & Annonaceae & Annona muricata L. & $\mathrm{N}$ & Guanábana & $A$ \\
\hline 7 & Annonaceae & Annona squamosa L. & $\mathrm{N}$ & Anona & A \\
\hline 8 & Annonaceae & $\begin{array}{l}\text { Polyalthia Iongifolia (Sonn.) } \\
\text { Thwaites }\end{array}$ & I & $\begin{array}{l}\text { Falsa ashoka, árbol de } \\
\text { buda, árbol indio mástil }\end{array}$ & A \\
\hline 9 & Annonaceae & $\begin{array}{l}\text { Sapranthus violaceus (Dunal) } \\
\text { Saff. }\end{array}$ & $\mathrm{N}$ & Ala de murciélago, urraco & $A$ \\
\hline 10 & Apocynaceae & Cascabela thevetia L. Lippold & $\mathrm{N}$ & Campanilla amarilla & A \\
\hline 11 & Apocynaceae & Allamanda cathartica L. & $\mathrm{N}$ & $\begin{array}{l}\text { Amor de estudiante, } \\
\text { bejuco de San José }\end{array}$ & $L$ \\
\hline 12 & Araceae & $\begin{array}{l}\text { Philodendron lacerum (Jacq.) } \\
\text { Schott }\end{array}$ & I & Mano de león & $H$ \\
\hline 13 & Araucariaceae & $\begin{array}{l}\text { Araucaria heterophylla (Salisb.) } \\
\text { Franco }\end{array}$ & I & Araucaria, pino australiano & A \\
\hline 14 & Arecaceae & $\begin{array}{l}\text { Acoelorraphe wrightii (Griseb. \& } \\
\text { H. Wendl.) H. Wendl. ex Becc. }\end{array}$ & $\mathrm{N}$ & Cubas, suyate & $H$ \\
\hline 15 & Arecaceae & $\begin{array}{l}\text { Acrocomia aculeata (Jacq.) Lodd. } \\
\text { ex Mart. }\end{array}$ & $\mathrm{N}$ & Coyol & $\mathrm{H}$ \\
\hline 16 & Arecaceae & Caryota mitis Lour. & I & Cola de pescado & $\mathrm{H}$ \\
\hline 17 & Arecaceae & Cocos nucifera L. & D & Coco, cocotero & $\mathrm{H}$ \\
\hline 18 & Arecaceae & $\begin{array}{l}\text { Dypsis lutescens (H. Wendl.) } \\
\text { Beentje \& J. Dransf. }\end{array}$ & I & Palma areca, areca & $H$ \\
\hline 19 & Arecaceae & $\begin{array}{l}\text { Roystonea regia (Kunth) O.F. } \\
\text { Cook }\end{array}$ & $\mathrm{N}$ & Palma real & $H$ \\
\hline 20 & Arecaceae & Washingtonia robusta $\mathrm{H}$. Wendl. & I & Palmera de abanico & $H$ \\
\hline 21 & Asparagaceae & Agave americana L. & । & Maguey & $\mathrm{H}$ \\
\hline 22 & Asparagaceae & $\begin{array}{l}\text { Asparagus densiflorus (Kunth) } \\
\text { Jessop }\end{array}$ & I & $\begin{array}{l}\text { Esparraguera plumosa, } \\
\text { cola de zorro }\end{array}$ & $\mathrm{H}$ \\
\hline 23 & Asparagaceae & $\begin{array}{l}\text { Chlorophytum comosum (Thunb.) } \\
\text { Jacques }\end{array}$ & I & Malamadre, lazo de amor & $\mathrm{H}$ \\
\hline
\end{tabular}




\section{No. Familia}

24 Asparagaceae

25 Asparagaceae

26 Asparagaceae

27 Asparagaceae

28 Asteraceae

29 Asteraceae

30 Asteraceae

31 Asteraceae

32 Asteraceae

33 Asteraceae

34 Asteraceae

35 Asteraceae

36 Asteraceae

37 Asteraceae

38 Asteraceae

39 Bignoniaceae

40 Bignoniaceae

41 Bignoniaceae

42 Bignoniaceae

43 Bignoniaceae

44 Bignoniaceae

45 Bignoniaceae

46 Bignoniaceae

47 Bignoniaceae

48 Bignoniaceae

49 Boraginaceae

50 Boraginaceae
Especie

Cordyline fruticosa (L.)A. Chev.

Furcraea cabuya Trel

Sansevieria guineensis (L.) Willd.

Yucca guatemalensis Baker

Cirsium mexicanum DC.

Eupatorium odoratum L.

Eupatorium purpureum $\mathrm{L}$.

Parthenium hysterophorus $\mathrm{L}$.

Pluchea carolinensis (Jacq.) G.

Don

Sphagneticola trilobata (L.) Pruski

Taraxacum officinale F.H. Wigg.

Verbesina guatemalensis B.L.

Rob. \& Greenm.

Verbesina turbacensis Kunth

Vernonia patens Kunth

Zinnia peruviana (L.) L.

Crescentia alata Kunth

Crescentia cujete L.

Godmania aesculifolia (Kunth)

Standl.

Jacaranda mimosifolia D. Don

Podranea ricasoliana (Tanfani)

Sprague

Pyrostegia venusta (Ker Gawl.) Miers

Spathodea campanulata P.

Beauv.

Tabebuia chrysantha (Jacq.) G.

Nicholson

Tabebuia rosea (Bertol.) DC.

Tecoma stans (L.) Juss. ex Kunth

Cordia alliodora (Ruiz \& Pav.) Oken

Cordia dentata Poir.

\section{Origen Nombre común Hábito} de San José

N Maguey

Lengua de suegra

$\mathrm{N}$ Flor de izote

N Cardo, cardo santo, punzaquedito

N Amargo, chirivito, crucito

$\mathrm{H}$ Joe-pye-weed

$\mathrm{N}$ Ajenjo

N Siguapate, salvia santa

$\mathrm{N}$

Cansabobo, miona, tres puntas

Diente de león, amargón

$\mathrm{H}$

$\mathrm{H}$

$\mathrm{H}$ león, pascua de monte

N Apazotillo, sucunán

$\mathrm{N}$ Estrella, margarita, mulata

$\mathrm{N}$ Jícaro, morro

$\mathrm{N}$ Jícara

N Cacho de novillo, quebracho, masicarán Jacaranda

Arbusto de pandora, trompeta

Bignonia de invierno, liana de juego, trompetero de naranja

Llama del bosque

N Cortés

N Macuelizo

San Andrés

N Laurel 


\section{No. Familia}

\section{Especie}

\section{Origen Nombre común}

Hábito

51 Boraginaceae
52 Boraginaceae
53 Boraginaceae

Ehretia latifolia Loisel.

Heliotropium indicum L.

Heliotropium macrostachyum (DC.) Hemsl.

54 Bromeliaceae

Tillandsia pruinosa Sw.

55 Bromeliaceae Tillandsia recurvata (L.) L.

56 Cannabaceae Trema micrantha (L.) Blume.

57 Cannaceae Canna indica $\mathrm{L}$.

58 Capparaceae Crataeva tapia L.

59 Casuarinaceae Casuarina equisetifolia L.

60 Celastraceae Semialarium mexicanum (Miers) Mennega

61 Combretaceae Combretum fruticosum (Loefl.) Stuntz

62 Combretaceae Terminalia catappa L.

63 Commelinaceae Tradescantia pallida (Rose) D.R. Hunt

64 Cupressaceae Cupressus lusitanica Mill.

65 Davalliaceae Nephrolepis biserrata (Sw.) Schott

66 Euphorbiaceae Acalypha spachiana Baill.

67 Euphorbiaceae Acalypha wilkesiana Müller.

68 Euphorbiaceae Cnidoscolus urens (L.) Arthur

69 Euphorbiaceae Codiaeum variegatum (L.) Rumph. exA. Juss.

70 Euphorbiaceae Euphorbia milii Des Moul.

71 Euphorbiaceae Euphorbia pulcherrima Willd. ex Klotzsch

72 Euphorbiaceae Euphorbia trigona Haw.

73 Euphorbiaceae Ricinus communis $\mathrm{L}$.

74 Fabaceae Acacia farnesiana (L.) Willd.

75 Fabaceae Acacia pennatula (Schltdl. \& Cham.) Benth.

76 Fabaceae Albizia adinocephala (Donn. Sm.) Britton \& Rose ex Record 77 Fabaceae Andira inermis (W. Wright) Kunth

$\mathrm{N}$ Tigüilote

$\mathrm{N}$ Malmus

$\mathrm{N}$ Borraja, cola de alacrán

$\mathrm{N}$

$\mathrm{N}$

N Gallito

N Capulín, capulín cuerito

I Bandera, bijao, platanillo

N Cachimbo, matasanillo, naranjillo

\section{Casuarina}

N Guaracaco, matapiojos, oreja de mono

N Papamiel

\section{Almedra}

Ala de cucaracha

N Ciprés

Manto de Jesús

Chichicaste

Cola de gallo, laurel

$\mathrm{H}$

Corona de Cristo

Pascua

$\mathrm{H}$

Árbol africano de leche,

H corona

$\mathrm{N}$ Ricinus, higuerilla $\mathrm{H}$

N Espino blanco, guarumo, subín

N Espino negro, carbón, carbón blanco

N Candelillo, gavilancillo, A madre de cacao

$\mathrm{N}$ Almendro de monte,
A

H

A

$\mathrm{H}$

$\mathrm{H}$

H

$\mathrm{H}$

$\mathrm{H}$

A

$\mathrm{H}$

H

$\mathrm{H}$

$\mathrm{H}$

A

$\mathrm{H}$

$\mathrm{H}$

A

A

.

A

A

H

A




\begin{tabular}{|c|c|c|c|c|c|}
\hline No. & Familia & Especie & Origen & Nombre común & Hábito \\
\hline & & ex DC. & & $\begin{array}{l}\text { almendro de río, guaca- } \\
\text { mayo }\end{array}$ & \\
\hline 78 & Fabaceae & Bauhinia divaricata L. & I & $\begin{array}{l}\text { Casco de vaca, casco de } \\
\text { venado, tres puntas }\end{array}$ & A \\
\hline 79 & Fabaceae & Caesalpinia pulcherrima (L.) Sw. & $\mathrm{N}$ & $\begin{array}{l}\text { Barbona, flor de fuego, } \\
\text { palo de flor }\end{array}$ & $A R$ \\
\hline 80 & Fabaceae & Coursetia polyphylla Brandegee & $\mathrm{N}$ & Chilincoco & AR \\
\hline 81 & Fabaceae & Crotalaria pumila Ortega & $\mathrm{N}$ & Sonito & $\mathrm{H}$ \\
\hline 82 & Fabaceae & Delonix regia (Bojer ex Hook.) Raf. & I & Morazán & $A$ \\
\hline 83 & Fabaceae & Desmodium nicaraguense Oerst. & $\mathrm{N}$ & & $H$ \\
\hline 84 & Fabaceae & $\begin{array}{l}\text { Enterolobium cyclocarpum (Jacq.) } \\
\text { Griseb. }\end{array}$ & $\mathrm{N}$ & Guanacaste & $A$ \\
\hline 85 & Fabaceae & Indigofera suffruticosa Mill. & $\mathrm{N}$ & Añil, barbasco, jiquilite & A \\
\hline 86 & Fabaceae & Inga vera Benth. & I & Guaba, paterna, guajiniquil & A \\
\hline 87 & Fabaceae & $\begin{array}{l}\text { Leucaena leucocephala (Lam.) de } \\
\text { Wit }\end{array}$ & I & Leucena, ipil-ipil & A \\
\hline 88 & Fabaceae & $\begin{array}{l}\text { Leucaena diversifolia (Schltdl.) } \\
\text { Benth. }\end{array}$ & I & Quebrachillo & A \\
\hline 89 & Fabaceae & $\begin{array}{l}\text { Lonchocapus sanctuarii Standl. \& } \\
\text { L.O. Williams }\end{array}$ & $\mathrm{N}$ & & $A$ \\
\hline 90 & Fabaceae & Lysiloma auritum (Schltdl.) Benth. & $\mathrm{N}$ & $\begin{array}{l}\text { Quebracho, quebracho } \\
\text { morroñoso }\end{array}$ & A \\
\hline 91 & Fabaceae & $\begin{array}{l}\text { Mimosa albida Humb. \& Bonpl. ex } \\
\text { Willd. }\end{array}$ & $\mathrm{N}$ & $\begin{array}{l}\text { Vergonzosa, zarza, zarza } \\
\text { hueca }\end{array}$ & $A R$ \\
\hline 92 & Fabaceae & Mimosa pudica L. & $\mathrm{N}$ & $\begin{array}{l}\text { Dormilona, puta vieja, } \\
\text { sensitiva }\end{array}$ & $\mathrm{H}$ \\
\hline 93 & Fabaceae & Mimosa tenuiflora (Willd.) Poir. & $\mathrm{N}$ & $\begin{array}{l}\text { Carbón, carbón blanco, } \\
\text { carbón comayagua }\end{array}$ & $A$ \\
\hline 94 & Fabaceae & Myrospermum frutescens Jacq. & I & Cereipo, guatamare & A \\
\hline 95 & Fabaceae & $\begin{array}{l}\text { Pithecellobium dulce (Roxb.) } \\
\text { Benth. }\end{array}$ & $\mathrm{N}$ & Mangollano, michigüiste & A \\
\hline 96 & Fabaceae & $\begin{array}{l}\text { Racosperma mangium (Willd.) } \\
\text { Pedley }\end{array}$ & I & & A \\
\hline 97 & Fabaceae & Samanea saman (Jacq.) Merr. & $\mathrm{N}$ & $\begin{array}{l}\text { Carreto, cenicero, palo } \\
\text { verde }\end{array}$ & A \\
\hline 98 & Fabaceae & $\begin{array}{l}\text { Senna pallida (Vahl) H.S. Irwin \& } \\
\text { Barneby }\end{array}$ & $\mathrm{N}$ & & A \\
\hline 99 & Fabaceae & $\begin{array}{l}\text { Senna reticulata (Willd.) H.S. Irwin } \\
\text { \& Barneby }\end{array}$ & $\mathrm{N}$ & $\begin{array}{l}\text { Sorocontil, tarantán, } \\
\text { zambrano }\end{array}$ & A \\
\hline
\end{tabular}




\begin{tabular}{|c|c|c|c|c|}
\hline Familia & Especie & Origen & Nombre común & Hábit \\
\hline 100 Heliconiaceae & Heliconia psittacorum L.f. & I & & \\
\hline 101 Heliconiaceae & Heliconia bihai (L.) L. & । & Bijao & \\
\hline 102 Hydrangeaceae & Hydrangea spp. & । & Hortensia & \\
\hline 3 Lythraceae & Punica granatum $\mathrm{L}$. & I & Granada & \\
\hline 104 Lythraceae & Lagerstroemia indica L. & I & Árbol de júpiter & $A$ \\
\hline 105 Lythraceae & Pehria compacta & $\mathrm{N}$ & (Rusby) Sprague & \\
\hline 106 Malpighiaceae & Byrsonima crassifolia (L.) Kunth & $\mathrm{N}$ & Nance & \\
\hline 107 Malvaceae & $\begin{array}{l}\text { Ceiba aesculifolia (Kunth) Britten } \\
\text { \& Baker f. }\end{array}$ & $\mathrm{N}$ & Ceibillo, pochote & \\
\hline 108 Malvaceae & Ceiba pentandra (L.) Gaertn. & $\mathrm{N}$ & Ceiba & \\
\hline 109 Malvaceae & Guazuma ulmifolia Lam. & $\mathrm{N}$ & Tapaculo & \\
\hline 110 Malvaceae & Hibiscus mutabilis $\mathrm{L}$. & I & Mar pacífico & $\mathrm{AF}$ \\
\hline 111 Malvaceae & Hibiscus rosa-sinensis L. & I & Rosa de china, hibisco & $A$ \\
\hline 112 Malvaceae & Sida acuta Burm. f. & $\mathrm{N}$ & $\begin{array}{l}\text { Malva de chancho, malva } \\
\text { murruca }\end{array}$ & \\
\hline 113 Malvaceae & Sterculia apetala (Jacq.) H. Karst. & $\mathrm{N}$ & $\begin{array}{l}\text { Cáñamo, cáñamo de } \\
\text { honduras, mano de león }\end{array}$ & \\
\hline 114 Malvaceae & Triumfetta lappula L. & $\mathrm{N}$ & $\begin{array}{l}\text { Agua de mecate, mozote, } \\
\text { mozotillo }\end{array}$ & $\mathrm{Al}$ \\
\hline 115 Malvaceae & Waltheria indica L. & $\mathrm{N}$ & $\begin{array}{l}\text { Escobilla, flor de cebra, } \\
\text { mozote de valle }\end{array}$ & \\
\hline 116 Melastomataceae & Tibouchina urvilleana (DC.) Cong. & I & Planta de la gloria & $A F$ \\
\hline 117 Meliaceae & Azadirachta indica A. Juss. & । & $\mathrm{Nim}$ & \\
\hline 118 Meliaceae & Cedrela odorata L. & $\mathrm{N}$ & Cedro & \\
\hline 119 Meliaceae & Melia azedarach L. & I & Árbol del paraíso & \\
\hline 120 Meliaceae & Swietenia humillis Zucc. & $\mathrm{N}$ & $\begin{array}{l}\text { Caoba, caoba de pacífico, } \\
\text { caobilla }\end{array}$ & \\
\hline 121 Meliaceae & Swietenia macrophylla King & $\mathrm{N}$ & $\begin{array}{l}\text { Caoba, caoba hondureña, } \\
\text { cóbano }\end{array}$ & \\
\hline 122 Moraceae & Ficus pertusa L.f. & $\mathrm{N}$ & Higo & \\
\hline 123 Moraceae & Ficus benjamina L. & । & Benjamina & \\
\hline 124 Moraceae & Ficus elastica Roxb. ex Hornem. & I & Árbol de caucho & \\
\hline 125 Moringaceae & Moringa oleifera Lam. & । & Moringa & \\
\hline 126 Muntingiaceae & Muntingia calabura L. & $\mathrm{N}$ & Capulín & \\
\hline 127 Myrtaceae & $\begin{array}{l}\text { Callistemon citrinus (Curtis) } \\
\text { Skeels }\end{array}$ & । & Árbol de cepillo & \\
\hline 128 Myrtaceae & Callistemon lanceolatus Sweet & । & Cepillo & \\
\hline $\begin{array}{l}129 \text { Myrtaceae } \\
130 \text { Myrtaceae }\end{array}$ & $\begin{array}{l}\text { Eucalyptus camaldulensis Dehnh. } \\
\text { Psidium guianense Pers. }\end{array}$ & । & $\begin{array}{l}\text { Eucalipto } \\
\text { Guayaba }\end{array}$ & \\
\hline
\end{tabular}


No. Familia

131 Myrtaceae

132 Myrtaceae

133 Myrtaceae

134 Nyctaginaceae

135 Nyctaginaceae

136 Papaveraceae

137 Phytolaccaceae

138 Picramniaceae

139 Pinaceae

140 Poaceae

141 Poaceae

142 Poaceae

143 Poaceae

144 Poaceae

145 Polygonaceae

146 Pontederiaceae

147 Proteaceae

148 Rosaceae

149 Rubiaceae

150 Rubiaceae

151 Rubiaceae

152 Rutaceae

153 Rutaceae

154 Salicaceae

155 Salicaceae
Especie

Psidum guajava $\mathrm{L}$.

Syzygium cumini (L.) Skeels

Syzygium jambos (L.) Alston

Boungainvillea spectabillis Willd.

Pisonia aculeata L.

Argemone mexicana L.

Phytolacca rivinoides Kunth \&

C.D. Bouché

Alvaradoa amorphoides Liebm.

Pinus oocarpa Schiede ex Schltdl.

Chloris radiata (L.) Sw.

Hyparrhenia rufa (Nees) Stapf

Melinis repens (Willd.) Zizka

Oplismenus hirtellus (L.) P. Beauv.

Urochloa maxima (Jacq.) R.D.

Webster

Ruprechtia salicifolia (Cham. \&

Schltdl.) C.A. Mey.

Eichhornia crassipes (Mart.)

Solms

Grevillea robusta A. Cunn. ex R. Br.

Eriobotrya japonica (Thunb.) Lindl.

Hamelia patens Jacq.

Ixora coccinea L.

Mussaenda erythrophylla

Schumach. \& Thonn.

Citrus limon (L.) Osbeck

Citrus $x$ sinensis (L.) Osbeck

Salix humboldtiana Willd.

Xylosma flexuosa (Kunth) Hemsl.

\section{Origen Nombre común}

Hábito

\section{Guayaba}

Jambolana, negrito

Manzana de castilla, manzana pedorra, manzana rosa

Napoleón

N Iscambrón blanco, uña de

AR gato

N Cardo santo

$\mathrm{N}$ Bledo de burro, mantete, quilete

N Cola de ardilla, limoncillo, A zorra

N Pino, ocote

N Paraguita blanco

Jaraguá, pasto jaraguá, zacate jaraguá

Flor moradita, pasto de seda, zacate rosado

Pasto de sombra, zacate $\quad \mathrm{H}$ perfumado

Guinea, pasto de guinea, zacate de guinea

Vivaró

Lirio de agua

Gravilea

Níspero zorrillo real

Bouquet, corona de la reina

Flor de trapo

Limón

Naranja

Sauce

N Aguja de ara, comida de

culebra, espina de sapo
H

A

A

AR

$\mathrm{H}$

A

A

A

A

$\mathrm{H}$

$\mathrm{H}$

$A$

A

$\mathrm{H}$

$\mathrm{H}$

$\mathrm{H}$

$H$

H

$A$

$\mathrm{H}$

A

A

A

A 


\begin{tabular}{|c|c|c|c|c|c|}
\hline No. & Familia & Especie & Origen & Nombre común & Hábito \\
\hline 156 & Sapindaceae & Melicoccus bijugatus Jacq. & I & $\begin{array}{l}\text { Escajocote, mamón, } \\
\text { mamoncillo }\end{array}$ & A \\
\hline 157 & Sapindaceae & Dodonaea viscosa Jacq. & I & Casco de venado, huesito & AR \\
\hline 158 & Scrophulariaceae & $\begin{array}{l}\text { Russelia equisetiformis Schltdl. \& } \\
\text { Cham. }\end{array}$ & I & Lágrima de cupido & $\mathrm{H}$ \\
\hline 159 & Simaroubaceae & Simarouba glauca DC. & $\mathrm{N}$ & Negrito, talchocote & A \\
\hline 160 & Solanaceae & Cestrum nocturnum L. & $\mathrm{N}$ & $\begin{array}{l}\text { Galán de noche, dama de } \\
\text { noche }\end{array}$ & $A R$ \\
\hline 161 & Solanaceae & Nicotiana glauca Graham & $\mathrm{N}$ & & $H$ \\
\hline 162 & Solanaceae & Solanum erianthum Dunal. & $\mathrm{N}$ & $\begin{array}{l}\text { Friegaplatos, hoja blanca, } \\
\text { tomate de espiga }\end{array}$ & $H$ \\
\hline 163 & Solanaceae & Solanum lanceolatum Ruiz \& Pav. & $\mathrm{N}$ & $\begin{array}{l}\text { Friegaplatos, tomatillo, uva } \\
\text { azul }\end{array}$ & $\mathrm{H}$ \\
\hline 164 & Solanaceae & Solanum rudepannum Dunal & $\mathrm{N}$ & $\begin{array}{l}\text { Tomate de montaña, } \\
\text { tomatillo }\end{array}$ & $\mathrm{H}$ \\
\hline 165 & Solanaceae & Solanum torvum Sw. & $\mathrm{N}$ & $\begin{array}{l}\text { Diente de perro, lavaplatos, } \\
\text { zarza }\end{array}$ & $\mathrm{H}$ \\
\hline 166 & Thelypteridaceae & Thelypteris patens (Sw.) Small & $\mathrm{N}$ & & $H$ \\
\hline 167 & Verbenaceae & Duranta erecta L. & N & Júpiter & $\mathrm{H}$ \\
\hline 168 & Verbenaceae & Petrea volubilis $\mathrm{L}$. & I & $\begin{array}{l}\text { Carbonera de monte, chapa- } \\
\text { rro, manto del nazareno }\end{array}$ & AR \\
\hline 169 & Verbenaceae & Lippia cardiostegia Benth. & N & $\begin{array}{l}\text { Palo de hoja blanca, vara } \\
\text { blanca }\end{array}$ & $\mathrm{H}$ \\
\hline 170 & Verbenaceae & Lantana camara L. & $\mathrm{N}$ & $\begin{array}{l}\text { Cinco negritos, comida } \\
\text { paloma, siete negritos }\end{array}$ & $\mathrm{H}$ \\
\hline 171 & Verbenaceae & $\begin{array}{l}\text { Stachytarpheta cayennensis } \\
\text { (Rich.) Vahl }\end{array}$ & $\mathrm{N}$ & $\begin{array}{l}\text { Cola de alacrán, cola de } \\
\text { ratón, mozote }\end{array}$ & $\mathrm{H}$ \\
\hline 172 & $\begin{array}{l}\text { Xanthor- } \\
\text { rhoeaceae }\end{array}$ & Aloe vera (L.) Burm.f. & I & Sábila & $\mathrm{H}$ \\
\hline
\end{tabular}

Origen N: nativo; I: introducida; hábito A: árbol; AR: arbusto; H: hierba; L: liana. 